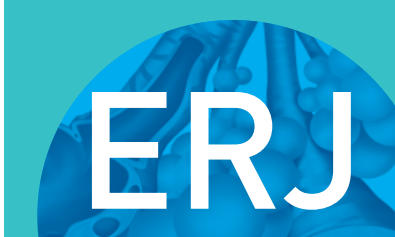

open research
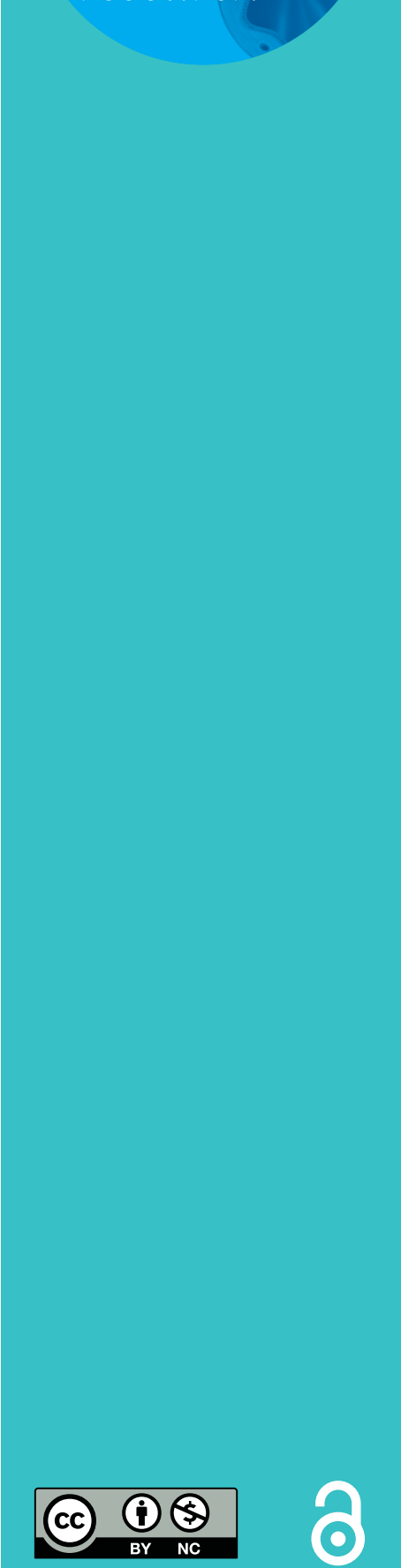

\title{
Targeted therapy in eosinophilic chronic obstructive pulmonary disease
}

\author{
Mathieu Fieldes (10), Chloé Bourguignon ${ }^{1}$, Said Assou ${ }^{1}$, Amel Nasri ${ }^{1}$, \\ Aurélie Fort ${ }^{2,3}$, Isabelle Vachier ${ }^{2}$, John De Vos ${ }^{1,4}$, Engi Ahmed ${ }^{2}$ and \\ Arnaud Bourdin (10) 2,3
}

Affiliations: ${ }^{1}$ IRMB, INSERM, Montpellier University Hospital, Montpellier, France. ${ }^{2}$ Dept of Respiratory Diseases, Montpellier University Hospital, INSERM, Montpellier, France. ${ }^{3}$ PhyMedExp, University of Montpellier, INSERM U1046, Montpellier, France. ${ }^{4}$ Dept of Cell and Tissue Engineering, Montpellier University Hospital, Montpellier, France.

Correspondence: Engi Ahmed, Hopital Arnaud de Villeneuve, 371 Av Doyen Gaston Giraud, 34295 Montpellier Cx 5, France. E-mail: e-ahmedachu-montpellier.fr.

ABSTRACT Chronic obstructive pulmonary disease (COPD) is a common and preventable airway disease causing significant worldwide mortality and morbidity. Lifetime exposure to tobacco smoking and environmental particles are the two major risk factors. Over recent decades, COPD has become a growing public health problem with an increase in incidence. COPD is defined by airflow limitation due to airway inflammation and small airway remodelling coupled to parenchymal lung destruction. Most patients exhibit neutrophil-predominant airway inflammation combined with an increase in macrophages and $\mathrm{CD}^{+} \mathrm{T}$-cells. Asthma is a heterogeneous chronic inflammatory airway disease. The most studied subtype is type 2 (T2) high eosinophilic asthma, for which there are an increasing number of biologic agents developed. However, both asthma and COPD are complex and share common pathophysiological mechanisms. They are known as overlapping syndromes as approximately $40 \%$ of patients with COPD present an eosinophilic airway inflammation. Several studies suggest a putative role of eosinophilia in lung function decline and COPD exacerbation. Recently, pharmacological agents targeting eosinophilic traits in uncontrolled eosinophilic asthma, especially monoclonal antibodies directed against interleukins (IL-5, IL-4, IL-13) or their receptors, have shown promising results. This review examines data on the rationale for such biological agents and assesses efficacy in T2-endotype COPD patients.

@ERSpublications

Patients with severe COPD and eosinophilic inflammation experience uncontrolled symptoms despite optimal pharmaceutical treatment. The development of new biomarkers is needed for better phenotyping of patients to propose innovative targeted therapy. https://bit.ly/2KzWuNO

Cite this article as: Fieldes M, Bourguignon C, Assou S, et al. Targeted therapy in eosinophilic chronic obstructive pulmonary disease. ERJ Open Res 2021; 7: 00437-2020 [https://doi.org/10.1183/ 23120541.00437-2020]. 


\section{Introduction}

Chronic obstructive pulmonary disease (COPD) is a growing cause of morbidity and mortality worldwide [1]. The Global Burden of Disease Study 2015 estimated approximately 174 million prevalent cases in the world. Over the last two decades, specialists in the field have noticed a significant increase in patient numbers mainly due to global population ageing and environmental factors [2]. Several studies report a high prevalence rate ranging from $2.1 \%$ to $26.1 \%$ of the adult population depending on age, sex, smoking habits, world region and study inclusion criteria used [2-8]. A higher prevalence is reported among men, but recent data indicate a progressive sex-ratio equilibration due to a rise in tobacco smoking in high-income countries and an increase in environmental exposures in low/middle-income countries [4, 9-13]. As underlined by the ELISABET (Enquête Littoral Souffle Air Biologie Environnement) study, considerable heterogeneity in the prevalence of COPD is due to an impressive $76.4 \%$ under-diagnosis rate [14].

In 2015, over 3 million people died from COPD in the world [2]. World Health Organization (WHO) projections are quiet alarming and highlight a possibility of 6 million deaths at the 2050 horizon [15]. Healthcare systems and society face a complex economic problem given that COPD is ranked eighth place in terms of disability-adjusted life years (DALYs), accounting for $2.6 \%$ of global DALYs [2]. In Europe, the total cost of respiratory diseases, including healthcare and productivity loss, represents more than EUR 380 billion per year, with EUR 48.4 billion being directly imputable to COPD [16]. Costs are clearly associated with exacerbation frequency, hospital admissions and disease severity [17-21]. Indeed, higher severity of COPD is significantly associated with a higher risk of death [22]. There is no curative treatment currently available and disease management is highly focussed on the symptomatic side and limitation of acute exacerbations. New studies based on the triple association of inhaled glucocorticoid (ICS), long-acting $\beta_{2}$-agonist (LABA) and long-acting muscarinic antagonist (LAMA) showed a significant $25 \%$ reduction in risk of moderate or severe exacerbations compared with $15 \%$ for dual therapy (ICS-LABA and LAMA-LABA) [23]. Emerging evidence highlights the complexity of the disease, with the existence of specific COPD patient endotypes and "treatable traits", such as predominant eosinophilic inflammation [24]. A targeted strategy adapted to the different treatable traits/endotypes appears more appropriate. Hence, anti-eosinophilic drugs developed for asthma bring new hope for patients with COPD. This review aims to discuss results from recently published data reporting evidence of efficacy in some patients with COPD.

\section{Pathophysiology and molecular mechanisms General pathophysiology}

COPD is a preventable disease clinically defined by persistent respiratory symptoms and airflow limitation during forced expiration mainly due to airway and/or alveolar abnormalities [12, 25]. Airflow limitation is due to an increased airway resistance combined with mucociliary clearance failure and progressive accumulation of mucus exudate in distal airway lumens [26].

A local chronic inflammatory response is combined with an abnormal and excessive airway remodelling subsequent to damage repair, leading in turn to an alteration of the epithelial barrier and a thickening of the conducting walls of distal airways $(<2 \mathrm{~mm}$ of diameter) $[27,28]$. In addition to lumen narrowing, a substantial decrease in distal airway numbers has been clearly correlated with the COPD severity grade [29]. Lifetime exposure to tobacco smoking and environmental particles (domestic biomass combustion and air pollution) appear to be the two major COPD risk factors in high-income and developing countries respectively $[30,31]$.

ATsou et al. [3] demonstrate a significant trend between COPD and the amount of tobacco smoking; people consuming over 30 pack-years have a median (interquartile range); 3.73 (2.62-5.29) higher risk of developing COPD $[4,5]$. Recently, emerging evidence suggests that accelerated forced expiratory volume in $1 \mathrm{~s}\left(\mathrm{FEV}_{1}\right)$ decline is just one of the possible disease trajectories. Paediatric roots are involved in more than half of COPD cases, where abnormal development and lung growth during childhood leads to an incomplete pulmonary function at the age of 20 [32-34]. New genome-wide association studies (GWAS) highlight the genetic background in COPD emergence [35-38] with $\alpha_{1}$-antitrypsin deficiency as one of the most well-described genetic disorders [39]. Historically the COPD inflammatory profile is mainly characterised by an increase in macrophages, neutrophils and $\mathrm{CD} 8^{+} \mathrm{T}$-cells in peripheral airways and lung parenchyma due to non-T-helper cell type 2 mechanisms, thus steroid non-responsive [40-43]. The percentage of neutrophils in the sputum appears to be higher in COPD patients, with more severe airflow obstruction and development of neutrophilic bronchitis during exacerbations [44]. A therapeutic strategy targeting this neutrophilic trait (anti-interleukin (IL)-8 and anti-CXCR2) leads to minimal reduction in inflammation with limited reduced blood neutrophil counts or clinical benefit [45]. SUN et al. [46] reported a significant variation in Th1/Th2 cytokines between acute exacerbation and remission of COPD. They demonstrated that the imbalance of cytokines secreted by Th1 and Th2 cells was disrupted in COPD 
patients. Indeed, acute COPD exacerbation was associated with a decrease in Th1 cells and a dominance by Th2 cells, with a normalisation of Th1 cell numbers during the remission step [46].

\section{COPD and the eosinophilic airway inflammation trait}

Eosinophils are terminally differentiated cells derived from $\mathrm{CD}_{3} 4^{+}$eosinophil-basophil progenitors in bone marrow. Progenitors undergo maturation under IL-5, IL-3, granulocyte-macrophage colony-stimulating factor (GM-CSF), IL-33 and thymic stromal lymphopoietin (TSLP) stimulation [47-50]. Eosinophils play pivotal roles in tissue homeostasis and the inflammatory processes harbouring pro-inflammatory genes that appear to be overexpressed in diseases such as asthma [51]. BAFADHEL et al. [40] reported from a 1 -year study a total of 182 exacerbations from 86 COPD cases, of which over one-quarter were associated with sputum eosinophilia. Variations in eosinophil counts according to treatment, exacerbation phase, and sampling time make it difficult to establish clear guidelines on the thresholds for defining eosinophilic inflammation in COPD. Indeed, there is no consensus definition for eosinophilic COPD [41, 49, 52, 53]. Currently, the cellular and molecular pathways leading to eosinophilic airway inflammation are well understood in asthma. Eosinophilic inflammation might not be identical in asthma and COPD. Two different pathogenic pathways of the adaptive or innate immune response are involved: i) Allergic eosinophilic inflammation driven by $\mathrm{CD}^{+}$Th2 lymphocytes and cytokines such as IL-4, IL-5 and IL-13. This phenotype is generally well controlled by ICS; ii) non-allergic eosinophilic inflammation probably imputable to IL-5 production by type 2 innate lymphoid cells (ILC2) [54, 55]. Eosinophilic inflammation is thought to be a main feature of asthma on a type 2-mediated airway inflammation background. COPD is known to be a highly heterogeneous disease with many different clinical features. Some patients present phenotypes differing to those from the general pathophysiology, for instance eosinophilic airway inflammation (eosinophilic endotype) [56-58]. Hence, some COPD and asthma patients share similar symptoms. This has been identified as the asthma-COPD overlap (ACO) [49, 59-61]. However, ACO has provided limited clinical and biological benefits regarding the management of chronic airway diseases given its relatively inaccurate diagnostic criteria (particularly the lack of clear-cut thresholds for quantitative parameters) and the heterogeneity of both inflammatory and structural change patterns [60].

It is noteworthy that a range of $20-40 \%$ COPD patients present an exacerbated eosinophilic profile in blood and/or sputum, not only during acute exacerbations but also in stable periods [40, 47, 62-65]. It has been demonstrated that blood eosinophil counts in COPD patients are associated with a higher frequency and severity of exacerbations [65-69]. Data from two multicentre and longitudinal cohorts (the ECLIPSE (Evaluation of COPD Longitudinally to Identify Predictive Surrogate End-points) and COPDGene studies) reported respective $22 \%$ and $32 \%(\mathrm{p}=0.006)$ increases in risk of exacerbation for COPD patients with a blood eosinophil count threshold of $\geqslant 300$ cells $\mu \mathrm{L}^{-1}$ [52]. A high number of eosinophils is also associated with a better response to ICS and could be a promising response biomarker $[66,67,70-72]$. Emerging transcriptomic evidence highlights only a small overlap between genes linked to blood eosinophilic inflammation in asthma and COPD [73]. However, the existence of an overlap is still debated. Considering for example the GLuCOLD (Groningen and Leiden Universities study of Corticosteroids in Obstructive Lung Disease) cohort; here the presence of a type 2 signature in the sputum related to a predicted eosinophilic pattern and steroid sensitivity outcome was not tracked by asthma history [74]. At a glance, it seems that type 2 traits and the subsequent eosinophilic patterns carry a significant genetic predisposition [75], whereas the clinical taxonomy plays a limited role and the overall story is far from being elucidated.

Over the last decade, emergence of specific anti-eosinophil molecules, such as monoclonal antibodies $(\mathrm{mAb})$ directed against IL-5, has led to major improvements in asthma control, improving lung function with diminution of exacerbations [76-79]. All these findings suggest that the eosinophilic inflammation pattern is a potential treatable trait in COPD patients. New evidence on this type 2 COPD endotype is rationale for the testing of drugs used for asthma treatment which selectively block eosinophilic and type 2 inflammation (figure 1).

\section{Anti-IL-5 therapies}

IL-5 is one of the major cytokines secreted by CD4 ${ }^{+}$Th2 lymphocytes, eosinophils, ILC2 cells, mastocytes, eosinophils, and basophils, in turn inducing the activation of multiple signalling pathways and the release of cytokines and chemokines [50]. These molecules have a pivotal role in eosinophil recruitment, activation, differentiation, proliferation and survival [80,81], and, in addition, eosinophil degranulation also has a major effect on airway inflammation [82]. Accordingly, IL-5 has become an interesting drug target in elevated eosinophil numbers among asthma patients. Targeted treatment, such as antibodies directed against the IL-5 cytokine (mepolizumab and reslizumab) or the IL-5 receptor (benralizumab), block eosinophil maturation. Note that the mechanism of action of benralizumab is different from that of mepolizumab and reslizumab, both binding exclusively to IL-5 and leading to a reduction in eosinophils. 


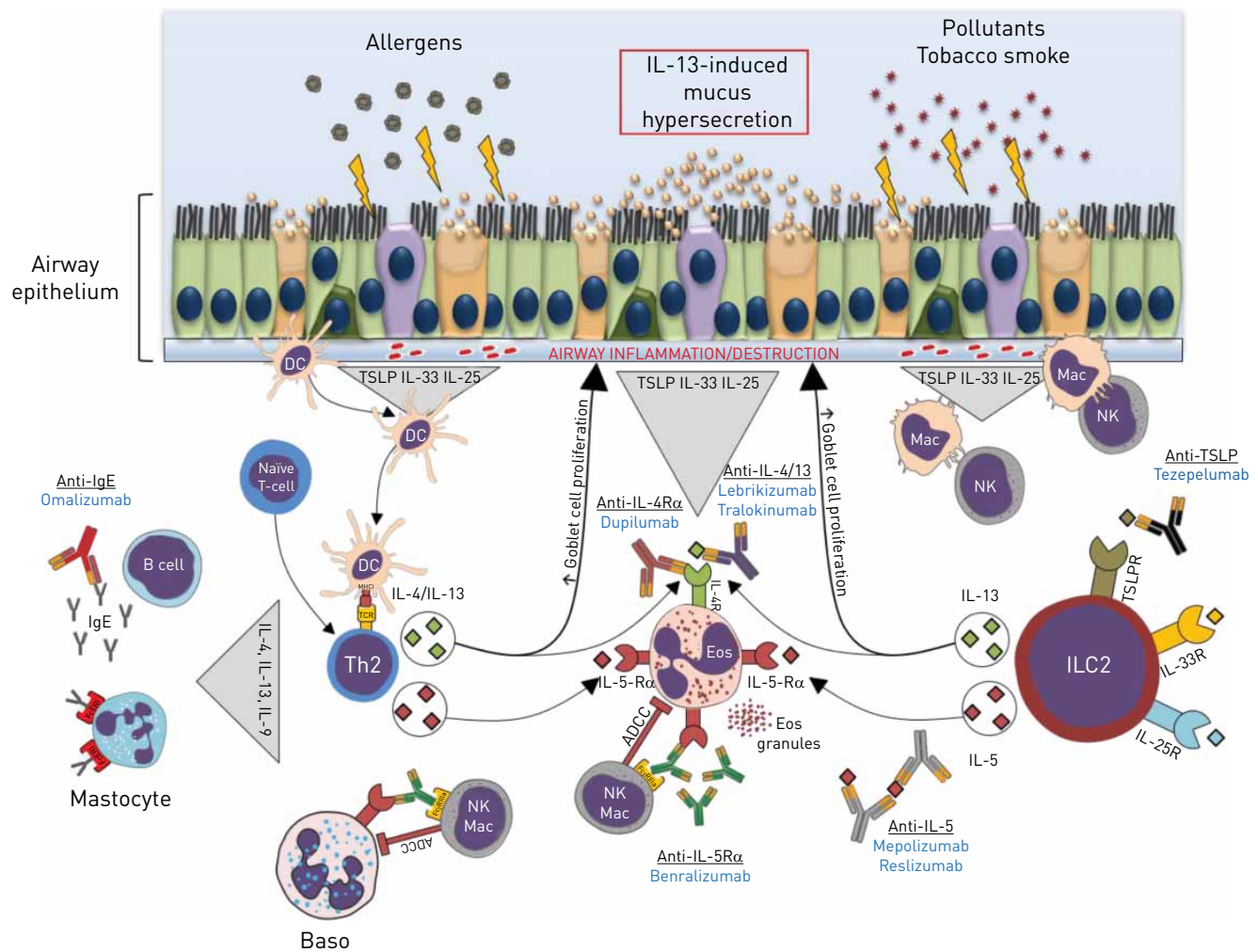

FIGURE 1 Simplified representation of the eosinophilic inflammation pattern in asthma and COPD. Allergens, cigarette tobacco, and other pollutants attack airway epithelial cells and contribute to a local injury. Release of epithelial-derived innate cytokines, interleukin (IL)-25, IL-33, and thymic stromal lymphopoietin (TSLP) in response to environmental factors play key roles in: i) the maturation of T-helper 2 (Th2) cells through dendritic cell activation; ii) the activation of innate immune cells including type 2 innate lymphoid cells. Release of Th2 cytokines (IL-4, IL-5 and IL-13) promotes the activation of resident macrophages and recruitment of innate cells such as basophils and eosinophils. Finally, activation of these several pathways participates in airway remodelling, mucus overexpression, and eosinophilic inflammation maintenance. Therapeutic strategy to control eosinophilic inflammation in asthma and COPD (monoclonal antibodies) are as follows. Benralizumab acts in an antibody-dependent cell-mediated cytotoxicity (ADCC) way resulting in eosinophilic depletion. Mepolizumab and reslizumab target the soluble IL-5 form to limit recruitment and activation of eosinophils. Omalizumab limits mastocyte activation through immunoglobulin (Ig)E depletion. Dupilumab inhibits eosinophil activation via IL-4R $\alpha$, contrary to lebrikizumab and tralokinumab which target soluble IL-13 cytokine. Tezepelumab blocks type 2 innate lymphoid cells (ILC2) activation by preventing TSLP binding. Baso: basophil; DC: dendritic cell; Eos: eosinophil; IL-5R $\alpha$ : IL-5 receptor $\alpha$; IL-25R: IL-25 receptor; IL-33R: IL-33 receptor; Mac: macrophage; MHCII: major histocompatibility complex class II; NK: natural killer cell; TCR: T-cell receptor. Illustrations adapted from Smart Servier under a Creative Commons Attribution 3.0 Unported (CC BY 3.0) license.

Thus, a strategy based on blood eosinophil depletion could play a role in the management of COPD.

\section{Mepolizumab}

Mepolizumab was approved in 2015 by the Food and Drug Administration (FDA) as an add-on therapy for the treatment of severe eosinophilic asthma [83, 84]. It is a fully-humanised mAb (immunoglobulin

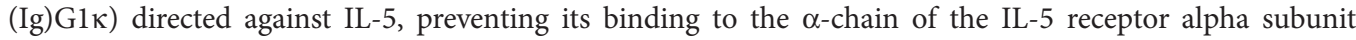
(IL-5R $\alpha$ ) present on the surface of eosinophils [85]. Pharmacokinetics is proportional to the dose and time independent. The half-life of mepolizumab is about 20 days, with a maximal concentration at 0.5 to $4.8 \mathrm{~h}$ after the beginning of perfusion [86]. The drug prevents the formation of the IL-5-receptor complex and blocks the activation of signalling pathways, leading to a limited eosinophil production, incomplete maturation and a decreased half-life [87].

ORTEGa et al. [88] reported from the DREAM (Mepolizumab for severe eosinophilic asthma) and MENSA (Mepolizumab as Adjunctive Therapy in Patients with Severe Asthma) studies a significant $47 \%$ reduction in mean exacerbation rate (rate ratio $=0.53, \mathrm{p}<0.0001$ ) in severe eosinophilic asthma. The authors also found a significant association between mepolizumab efficacy and high baseline blood eosinophil counts $\left(\geqslant 150\right.$ cells $\left.\mu \mathrm{L}^{-1}\right)$ [88]. A meta-analysis of eight different studies enrolling 1707 participants with 
severe asthma and high eosinophil levels concluded an improvement in quality of life and a reduction in asthma attacks without significant benefits on lung function [89].

DASGUPTA et al. [90] performed a single-centre, double-blind, randomised, placebo-controlled trial for 6 months including 18 patients of $40-80$ years old with moderate-to-severe COPD. The authors assessed the effect of monthly injections of mepolizumab $750 \mathrm{mg}$ or placebo in cigarette smoke-related COPD patients with persistent sputum eosinophilia. The primary objective was to determine if mepolizumab induced a significant reduction in sputum eosinophil count. Secondary outcomes were the assessment of the effects on blood eosinophil count, lung function, exacerbation rate, airway remodelling, symptoms and quality of life. Baseline sputum eosinophil counts represented $11 \%$ and $7.4 \%$ for the mepolizumab-treated arm $(n=8)$ and the placebo group $(\mathrm{n}=10)$ respectively. Baseline blood eosinophil counts were $0.7 \pm 0.5 \mathrm{cells} \cdot \mathrm{mm}^{-3}$ for the mepolizumab-treated and $0.33 \pm 0.29$ cells $\cdot \mathrm{mm}^{-3}$ for the placebo groups. After 6 months, a significant reduction in sputum $(0.50$ versus $2.20 \%, \mathrm{p}<0.05)$ and blood eosinophil counts $(0.03$ versus $0.26 ; \mathrm{p}<0.05)$ were reported in the mepolizumab-treated group. However, the additional secondary outcomes showed no significant changes; there was no improvement in lung function or exacerbation rates [90].

Two large cohort studies have also been carried out to assess anti-IL-5 efficacy on patients with moderate-to-severe exacerbations despite an adequate triple inhaled therapy composed of a combination of LABA, LAMA and ICS. These two phase III studies were the METREX (Mepolizumab versus Placebo as Add-on Treatment for Frequently Exacerbating COPD Patients) study and the METREO (Mepolizumab versus Placebo as Add-on Treatment for Frequently Exacerbating COPD Patients Characterised by Eosinophil Level) study. Both the METREX and METREO trials assessed the efficacy and safety of mepolizumab compared to placebo in patients with COPD and eosinophilic phenotype [91]. The primary outcome for both studies was the annual rate of moderate-to-severe exacerbations. The METREX study included patients with either an eosinophilic phenotype $\left(\geqslant 150\right.$ eosinophils $\cdot \mathrm{mm}^{-3}$ at screening or $\geqslant 300$ in the previous year) or a non-eosinophilic phenotype, contrary to the METREO study in which patient selection was based on blood eosinophil count. Treated patient groups consisted of mepolizumab $100 \mathrm{mg}$ (METREX and METREO) or $300 \mathrm{mg}$ (METREO) compared with placebo in addition to ICS/LABA/ LAMA tritherapy every 4 weeks for 52 weeks. Subcutaneous injection of mepolizumab $100 \mathrm{mg}$ once a month for 52 weeks was associated to a lower annual rate of moderate or severe exacerbations only in COPD patients with eosinophilic phenotype (high stratum group) in the METREX study [92, 93]. In this study, exacerbation rates of 1.40 versus 1.71 per year were observed in the mepolizumab-treated group compared to the placebo group respectively (rate ratio $=0.82, \mathrm{p}=0.04$ ). No significant improvements in the overall cohort or impact on emergency department visits were noted. On the contrary, the METREO study did not give any insight into the efficacy on exacerbation rate, at either the $100 \mathrm{mg}$ (rate ratio $=0.80$, $\mathrm{p}=0.07$ ) or $300 \mathrm{mg}$ (rate ratio=0.86, $\mathrm{p}=0.14$ ) doses [94]. However, both studies reported a well-tolerated mepolizumab treatment with similar incidence of adverse events compared to placebo groups. A dose-response relationship between the increase in eosinophil number and treatment efficacy has already been suggested [56]. Such hopeful results need to be discussed given that further increases in eosinophil count can lead to loss of asthma control after cessation of mepolizumab treatment [95-97]. Overall, the results of these studies are disappointing and contradictory. Stronger evidence of eosinophilic inflammation is now required for the enrolment of patients in the new phase III study MATINEE (Mepolizumab as Add-on Treatment In Participants With COPD Characterized by Frequent Exacerbations and Eosinophil Level; www.clinicaltrials.gov/ct2/show/NCT04133909). This means that the proportion of eligible patients will be smaller than expected. Interestingly, a GWAS performed on patients with COPD and blood hyper-eosinophilia did not demonstrate any robust associations between genetic variants and mepolizumab efficacy (biomarker efficacy) [98].

\section{Reslizumab}

Reslizumab is a humanised mAb (IgG4 $\mathrm{m}$ ) directed against IL-5 and prevents IL-5 binding at the eosinophil surface similarly to mepolizumab [99]. Plasma concentrations are dose-proportional with a maximal peak concentration obtained at $6.9 \mathrm{~h}$ after dosing and a reported half-life ranging between 24.5 to 30.1 days [100]. A study with patients aged 18-75 years old with uncontrolled eosinophilic asthma were randomly assigned and received monthly injections of reslizumab at $3 \mathrm{mg}^{-1} \mathrm{~kg}^{-1}(\mathrm{n}=53)$ or placebo $(\mathrm{n}=53)$ for 12 weeks. CASTRO et al. [101] reported the absence of a significant reduction in exacerbations $(\mathrm{p}=0.0833)$, but a significant improvement of $0.24 \mathrm{~L}$ in $\mathrm{FEV}_{1}$ parameter in the reslizumab group ( $\left.\mathrm{p}=0.0023\right)$. A significant diminution in eosinophils in the induced sputum $(p=0.0068)$ and in blood counts $(p<0.0001)$ was also observed [101]. To our knowledge, reslizumab has not yet been evaluated in COPD [41].

\section{Benralizumab}

Benralizumab is a humanised $\mathrm{mAb}$ directed against IL-5R $\alpha$ with an 18-day terminal half-life [102]. Excision of the fucose sugar residue in the $\mathrm{CH} 2$ region of the antibody (afucosylated antibody) results in a 
five- to 50-fold higher affinity for the Fc $\gamma$ receptor (human Fc $\gamma$ RIIIa) expressed on natural killer (NK) cells and macrophages. This modification leads to a 1000-fold increase in antibody-dependent cell-mediated cytotoxicity (ADCC) functions [103] and activation of this ADCC mechanism induces rapid eosinophil depletion [104-106].

Patients under benralizumab treatment with severe and uncontrolled eosinophilic asthma have shown a significant reduction in annualised exacerbation rate, an improvement in pre-bronchodilator $\mathrm{FEV}_{1}$, and a decrease in oral corticosteroid use [107-109]. A phase II, double-blind, randomised, controlled study was performed on an uncontrolled asthma cohort split into two groups: eosinophilic $(\mathrm{n}=324)$ and non-eosinophilic $(\mathrm{n}=285)$ phenotypes. At week 52 , the authors demonstrated a reduction in asthma exacerbations in the patients treated with benralizumab doses at $20 \mathrm{mg}(\mathrm{p}=0.019)$ and $100 \mathrm{mg}(\mathrm{p}=0.010)$. In particular, these patients had baseline blood eosinophil counts of at least 300 cells $\mu \mathrm{L}^{-1}[110]$. On the contrary, a phase II, double-blind, randomised, placebo-controlled study assessed the effect of benralizumab in COPD in 101 adults aged 40-85 years old with a moderate-to-severe disease and a sputum eosinophil count of $\geqslant 3 \%$. The primary outcome was annual rate of moderate-to-severe exacerbations of COPD at week 56. Patients received a placebo or a benralizumab $100 \mathrm{mg}$ injection, three doses every 4 weeks, followed by five doses every 8 weeks after 48 weeks. The placebo group reported a 0.92 (95\% CI $0.67-1.25$ ) and the benralizumab group a 0.95 (95\% CI $0.68-1.29$ ) annual rate of acute exacerbations, meaning in all that BRIGHTLING et al. [111] demonstrated a non-significant reduction of $3 \%$ (95\% CI -58-33; p=0.94) in exacerbations. On the other hand, benralizumab treatment was associated with a rapid diminution in blood and sputum eosinophils in patients with COPD and incidence of adverse events was similar to the placebo group. A significant improvement in post-bronchodilator $\mathrm{FEV}_{1}$ in the benralizumab arm was noted as similar to the placebo arm $(p=0.014)$ [111]. Despite this $\mathrm{FEV}_{1}$ increase, no difference was observed in health status [45]. GALATHEA (Benralizumab Efficacy in Moderate-to-Very Severe Chronic Obstructive Pulmonary Disease with Exacerbation History; n=1044) and TERRANOVA (Efficacy and Safety of Benralizumab in Moderate-to-Very Severe Chronic Obstructive Pulmonary Disease with Exacerbation History; $\mathrm{n}=1392$ ) were two phase III, double-blind, randomised, placebo-controlled trials. The primary endpoint was to assess the effect of benralizumab on COPD exacerbation rate. Patients of 40-85 years old were assigned in a 2:1 ratio into an eosinophilic $\left(\geqslant 220 \mathrm{cells} \cdot \mathrm{mm}^{-3}\right)$ or non-eosinophilic $\left(<220\right.$ cells $\left.\cdot \mathrm{mm}^{-3}\right)$ group. The first three doses were injected every 4 weeks then every 8 weeks, with final assessment at week 56. In both studies patients randomly received placebo or benralizumab at 30 or $100 \mathrm{mg}$. In the TERRANOVA study, an additional group of patients received benralizumab $10 \mathrm{mg}$. Results of the GALATHEA study showed no significant improvement in annual rate ratios for exacerbations at any treatment dose: benralizumab $30 \mathrm{mg}(0.96 ; \mathrm{p}=0.65)$ and benralizumab $100 \mathrm{mg}(0.83 ; \mathrm{p}=0.05)$. The same trend was detected in the TERRANOVA study, with corresponding rate ratios of $0.85(\mathrm{p}=0.06), 1.04$ $(p=0.66)$ and $0.93(p=0.40)$ in the $10 \mathrm{mg}, 30 \mathrm{mg}$ and $100 \mathrm{mg}$ benralizumab groups, respectively. No dose effect on benralizumab efficacy was detected and similar adverse events were observed. Interestingly, in both studies a moderate depletion of blood eosinophils was reported [112]. CRINER et al. [113] identified, from the TERRANOVA and GALATHEA trails, a subtype of patients characterised by i) a baseline blood eosinophil count $\geqslant 220$ cells $\mu \mathrm{L}^{-1}$; ii) three or more exacerbations in the previous year; and iii) tritherapy as best responder treatment in combination with benralizumab therapy. These patients were associated with a significant reduction in exacerbation rate ratio of 0.70 (95\% CI 0.56-0.88) under benralizumab $100 \mathrm{mg}$ treatment every 8 weeks compared with placebo. No improvement was reported for patients treated with benralizumab $30 \mathrm{mg}$ [113].

Altogether, these results are more disappointing than those observed with mepolizumab treatment, even more so given there was no relationship found with the level of eosinophilia. A new phase III study (www. clinicaltrials.gov/ct2/show/NCT01914757) will be relevant for the follow-up of the subset of patients who seemingly responded [113]. However, it is highly likely that if outcomes are yet again not achieved, benralizumab treatment will be ruled out for the treatment of COPD and the overall concept of eosinophilic COPD will be challenged.

To conclude, the European Medicines Agency declares that benralizumab is effective in patients with eosinophilic COPD, whereas the FDA claims there is not robust evidence supporting this.

Anti-IL-13/anti-IL-4 therapy

IL-4 and IL-13 Th2 cytokines are responsible for many functions and are involved in asthma and COPD development [114-118]. IL-13 binding to the IL-13 receptor alpha 1 (IL-13R $\alpha 1$ ) induces recruitment of IL-4 receptor alpha 1 (IL-4R $\alpha 1$ ), in turn forming a heterodimeric receptor complex responsible for the activation of signalling pathways $[114,119]$. IL-4 and IL-13 share similar biological effects, mainly as they bind the same receptor composed of IL-4R $\alpha 1$ and IL-13R $\alpha 1$, both expressed in airway epithelium [115, 120-122]. Indeed, IL-4 activates not only through signalling pathways via the IL-4R $\alpha$ and IL-13R $\alpha$ chains, but also via 
common gamma chain. Likewise, IL-13 has also been found to use IL-4R $\alpha$ and IL-13R $\alpha$ chains $[123,124]$. IL-13 is produced by $\mathrm{T}$ cells, mast cells, basophils and dendritic cells. It is involved in regulation of inflammatory and immune responses as well as mucous hypersecretion $[125,126]$. Eosinophils have shown secretion of IL-13 under GM-CSF and/or IL-5 stimulation [127] and ILC2 cells are also able to secrete IL-5 and IL-13 under stimulation of IL-33 and IL-25 [128]. ILC2s have been found increased in patients with stable COPD or during acute exacerbation [46].

\section{Dupilumab}

Dupilumab is a human mAb targeting IL-4R $\alpha$ leading to inhibition of IL-13 and IL-4 signalling [129]. A randomised, placebo-controlled, phase IIb clinical trial showed a significant increase in $\mathrm{FEV}_{1}$ parameter and a reduction in the rate of severe exacerbations in patients with uncontrolled asthma under dupilumab treatment. Improvements were consistent in two different treatment groups; dupilumab 200 or $300 \mathrm{mg}$ every 2 weeks regardless of baseline eosinophil count [130]. These encouraging data must be transposed to COPD patients. The BOREAS (A Pivotal Study to Assess the Efficacy, Safety and Tolerability of Dupilumab in Patients With Moderate-to-severe COPD With Type 2 Inflammation) study is underway (NCT03930732) and will give some insights for patients with COPD.

\section{Lebrikizumab}

Lebrikizumab is a humanised mAb that binds to soluble IL-13 and blocks activation of IL-4R $\alpha$ and IL-13R $\alpha 1$ heterodimers. Two studies related to lebrikizumab are LUTE and VERSE; double-blind, randomised, placebo-controlled studies enrolling 463 patients of 18-75 years old. HANANIA et al. [131] found an improvement in asthma exacerbation rate and lung function in patients with moderate-to-severe asthma and a high periostin profile [131]. A phase II, double-blind, randomised, placebo-controlled trial for lebrikizumab (NCT02546700) treatment has been recently carried out in patients with frequent COPD exacerbations despite ICS and at least one long-acting bronchodilator inhaler medication. Data are not yet available.

\section{Tralokinumab}

Tralokinumab is also a mAb which specifically targets IL-13 [126]. PIPER et al. [132] noted no significant improvement in asthma symptoms following treatment based on the Asthma Control Questionnaire score (ACQ-6; p=0.375), but a small effect on $\mathrm{FEV}_{1}$ was shown. Marone et al. [118] concluded a putative efficacy of tralokinumab in a highly selected cohort of asthmatics with an overexpression of IL-13. The authors concluded a minor role of IL-13 in severe asthma exacerbations [133]. No study or clinical trial data are available in patients with COPD.

Other targeting strategies: anti-TSLP, anti-IL-33, anti-IL-25 and anti-IgE drugs

Damage to airway epithelial cells induces the release of several cytokines, such as IL-33, IL-25 and TSLP, leading to eosinophilic inflammation through ILC2 and Th2 pathways [129]

\section{Tezepelumab}

Human TSLP is involved in activation of dendritic cells [134]. Activated DCs then induce conversion of $\mathrm{CD}^{+}$T-cells into Th2 cells able to produce the Th2 cytokines IL-4, IL-5, and IL-13 [135]. Tezepelumab is a human monoclonal antibody directed against TSLP, thus preventing its interaction with the TSLP receptor. CoRREN et al. [136] reported a significant $62 \%, 71 \%$ and $66 \%(\mathrm{p}<0.001$ for all comparisons with placebo group) diminution of annualised asthma exacerbation rates for tezepelumab treatment at $70 \mathrm{mg}$ every 4 weeks, $210 \mathrm{mg}$ every 4 weeks, or $280 \mathrm{mg}$ every 2 weeks respectively. Pre-bronchodilator $\mathrm{FEV}_{1}$ was also slightly higher in all tezepelumab-treated groups independently of blood eosinophil counts at the beginning of the study [136]. Currently, COURSE (Tezepelumab COPD Exacerbation Study) is a phase IIa, multicenter, double-blind, randomised trial (NCT04039113) recruiting patients to assess the efficacy of tezepelumab on moderate or severe COPD exacerbation rate ratios.

\section{Anti-lgE therapy}

Omalizumab

IgE plays an important role in allergic asthma [137]. Allergen-specific IgE binds to Fc receptors (FceRI) on the surface of mast cells, basophils and eosinophils. This binding induces allergic reactions through the release of inflammatory molecules [138]. Omalizumab is a recombinant humanised anti-IgE $\mathrm{mAb}$ indicated in patients with moderate-to-severe allergic asthma. Omalizumab binds to the Fc region of the IgE antibody, preventing the binding of IgE to high-affinity IgE receptors, and so blocking the signalling pathways responsible for the release of inflammatory mediators. Omalizumab has been shown to limit asthma exacerbation rates and annual rates of hospital admissions [139, 50]. MaLtBy et al. [140] reported an improvement in health-related quality of life in individuals with severe allergic asthma and ACO. 
The PROSPERO (Omalizumab in the Prospective Observational Study to Evaluate Predictors of Clinical Effectiveness in Response to Omalizumab) study is a multicentre, single-arm, prospective, 48-week observational study. Study analysis by Hanania et al. [141] showed similar improvements in asthma outcome among patients with and without ACO, but preserved lung function was reported in the ACO group.

\section{Discussion}

Eosinophilic airway inflammation is a common trait in patients with asthma and COPD. Some patients with COPD and uncontrolled asthma, despite maximal adequate treatment (ICS, LABA and LAMA), are eligible for treatment with molecules targeting specific components of eosinophilic inflammation in asthma. Mepolizumab has shown encouraging results in the asthma trajectory and has become one of the most studied anti-IL-5 therapies in COPD [142]. Regarding results from the METREX/METREO studies, mepolizumab therapy is thought to reduce the rate of exacerbations in COPD patients with an elevated blood eosinophil level. This trend was only confirmed in the METREX cohort, where patients with hyper-eosinophilia (high stratum) demonstrated a significant reduction in moderate-to-severe exacerbations. No significant trend was found in the METREO cohort regardless of the injection dose (100 or $300 \mathrm{mg}$ ). Patients with $\geqslant 300$ eosinophils. $\mathrm{mm}^{-3}$ benefit more from mepolizumab $100 \mathrm{mg}$ therapy, with a $23 \%$ lower mean annual rate of moderate or severe exacerbations (rate ratio=0.77 (95\% CI $0.63-0.94)$ ). It is noteworthy that the DREAM/MENSA trial in asthma reported a significant reduction in the mean exacerbation rate of patients under mepolizumab treatment (rate ratio $=0.53$ (95\% CI 0.44-0.62)) [88]. These differences could be explained by higher exacerbation rates in COPD and a weaker impact of IL-5 in the pathophysiology of COPD compared to asthma. Moreover, a decrease in sputum and blood eosinophil counts did not lead to significant improvements in lung function parameters or remodelling patterns, thus questioning the importance of eosinophil involvement in the disease [90]. Regarding the limited proof of efficacy and the cost-effectiveness balance, in 2018, the FDA decided not to approve mepolizumab as an add-on therapy for COPD [56]. No other anti-IL-5 therapies, such as reslizumab use, have been reported in COPD.

Direct targeting of IL-5R $\alpha$ with benralizumab in the GALATHEA and TERRANOVA trials has reported no significant improvement in annualised COPD exacerbation rate combined with limited diminution of blood eosinophil counts. The authors explain the difference due to different cut-off values for the eosinophil counts, limited patient asthma history characteristics, and variation in previous medications [112]. A subtype of patients in the GALATHEA and TERRANOVA trials with moderate-to-very severe COPD showed elevated peripheral blood eosinophils $\left(\geqslant 300\right.$ cells $\left.\mu \mathrm{L}^{-1}\right)$ and experienced more than three exacerbations. This was despite patient triple therapy being associated to a significant reduction in exacerbations. Heterogeneous results along with no reduction in acute COPD exacerbations, contrary to results with mepolizumab treatment, were possibly due to small sample sizes. In addition, no attenuation of symptoms nor impact on quality of life was reported, but surprisingly a significant lung function improvement based on $\mathrm{FEV}_{1}$ parameter was noted with long-lasting effects [111]. The RESOLUTE (Efficacy and Safety of Benralizumab in Moderate to Very Severe Chronic Obstructive Pulmonary Disease (COPD) With a History of Frequent Exacerbations) trial (NCT04053634) designed to assess the efficacy and safety of benralizumab in highly exacerbated patients with moderate-to-severe COPD will bring new insights on highly selected populations. The limited benefits of anti-IL-5/IL-5R treatment in COPD may relate to different factors: a) patient heterogeneity in clinical trials; currently applied cut-off values for blood eosinophil counts in COPD are less consensual than in asthma, meaning that the population may not be sufficiently enriched; b) mechanisms of airway eosinophilia in COPD might be different from asthma, driven in an IL-5-independent manner. For instance, eotaxin, GM-CSF, IL-13, impaired macrophage efferocytosis [143], CCL5 and alarmins, etc, have been shown to be potentially relevant candidates; or c) ambiguous clinical evidence that eosinophilia is differentially linked to COPD exacerbation, steroid sensitivity, or lung function compared to asthma.

It is not clear whether patients with sputum or blood eosinophilia represent a stable COPD phenotype over time. Little is known about the other clinical characteristics of T2 phenotype in COPD patients [144]. Whether eosinophilic airway inflammation arises due to increased bone marrow production and/or increased eosinophil recruitment into the airway is less well-documented than in asthma [145].

In a recent study [73], the authors aimed to identify the transcriptomic signatures in bronchial brushing samples from both patients with asthma and COPD in the U-BIOPRED (Unbiased BIOmarkers in PREDiction of respiratory disease outcomes) and EvA (Epidemiology of Vascular Ageing) cohorts, respectively. Using a blood eosinophil count cut-off of 200 cells $\cdot \mathrm{mL}^{-1}$, no genes were found differentially expressed between the COPD and asthma cohorts. The authors found that only 12 genes were associated with blood eosinophil count in the COPD cohort, versus more than 1000 among patients with asthma. These genes were in majority related to type 2-mediated immunity. The only common gene to both eosinophilic asthma and COPD was cystatin-SN (CST1). However, in the validation cohorts, CST1 and 
blood eosinophil count were weakly correlated. Cystatin is a cysteine protease inhibitor expressed by the airway and nasal epithelium and implicated in type 2 immunity, such as eosinophilic nasal polyps [146]. Epithelial CST1 expression is upregulated by the epithelial alarmins TSLP and IL-33, and it also stimulates alarmin release itself. Cystatin can also promote eosinophilic inflammation via fibroblast activation and subsequent release of pro-eosinophilic chemokines [146]. This study highlighted very few shared biological mechanisms between eosinophilic COPD and eosinophilic asthma. Unbiased bronchial epithelial gene expression studies have shown that CCL26 is also associated with blood eosinophil counts in COPD patients [74].

Studies based on the IL-4R receptor targeting strategy with dupilumab have shown significant improvement in asthmatic patient lung function combined with a diminution of annualised exacerbation rate [130, 147-149]. These encouraging results have led to the assessment of the use of dupilumab in COPD and a trial is ongoing (NCT03930732). Given the disappointing and disruptive results that have been reported from studies on the anti-IL-13 targeted strategy in uncontrolled and severe asthma, some authors have concluded a minor role of IL-13 in asthma [133]. In COPD, there may be eosinophil-driven mechanisms that may involve non-IL-5T2 cytokines such as IL-13, and therefore anti-IL-5 biologics do not show impressive clinical results. Eosinophil-derived IL-13 was shown to promote alveolar macrophage MMP-12 production and lead to airspace enlargement, indicating IL-13 involvement in the emphysematous progression of COPD [150].

Increased expression of CST1 and IL13 genes have been recently shown in eosinophilic COPD airways [151].

These promising sub-studies deduce the hypothesis that a small proportion of patients with COPD may benefit from anti-IL-5 therapy. Indeed, at this point most clinical trial evidence does not support the use of anti-IL-5 treatment in COPD [94, 112]. To our knowledge, no results on patients with COPD have been published for anti-IL-13 and anti-IL-4 treatment. Omalizumab, an anti-IgE treatment, has shown an improvement in health-related quality of life in individuals with severe allergic asthma and ACO. Anti-TSLP strategies are ongoing, with assessment in patients with COPD in a phase IIa, multicentre, double-blind randomised trial (NCT04039113) (COURSE study).

\section{Conclusions}

A significant proportion of patients with severe COPD and eosinophilic inflammation experience uncontrolled symptoms despite an optimal pharmaceutical treatment. Recently, targeted strategies directed specifically against cytokines or receptors involved in eosinophilic inflammation have provided significant improvement in asthma. Biological agents used in asthma have limited therapeutic effects on patients with COPD. These disappointing results are thought to be due to the existence of multiple disease origins and a highly complex role of eosinophils. Indeed, several studies have shown the global effects of eosinophilic patterns on exacerbations, but no impacts on the trajectory of the disease, such as lung function decline, were mentioned. A better understanding of such complex cellular mechanisms and a clear consensus on peripheral blood eosinophils are needed to improve patient gradations in routine clinical practice. Not only biomarkers but also elucidation of the role of eosinophilic and type 2 inflammation in COPD is warranted. In conclusion, the development of new biomarkers is mandatory for a better patient selection in order to propose these innovative therapies to the best responder patient profile. This step forward to personalised medical treatments for patients with COPD will match the right targeted treatment to the right patient.

Author contributions: All authors have read and agreed to the published version of the manuscript.

Conflict of interest: M. Fieldes has nothing to disclose. C. Bourguignon has nothing to disclose. S. Assou has nothing to disclose. A. Nasri has nothing to disclose. A. Fort has nothing to disclose. I. Vachier has nothing to disclose. J. De Vos has nothing to disclose. E. Ahmed has nothing to disclose. A. Bourdin reports grants, personal fees, nonfinancial support and other support from AstraZeneca, Boehringer Ingelheim, GlaxoSmithKline and Novartis; personal fees and nonfinancial support from Teva; personal fees, nonfinancial support and other support from Regeneron, Chiesi Farmaceuticals and Actelion; personal fees from Gilead; nonfinancial support and other support from Roche; and other support from Nuvaira, all outside the submitted work.

\section{References}

1 IMHE, Institute for Health Metrics and Evaluation (IHME). GBD Compare. Seattle, WA: IHME, University of Washington, 2015. http://www.healthdata.org/data-visualization/gbd-compare Date last accessed: May, 2020; date last updated: May 2020.

2 GBD 2015 Chronic Respiratory Disease Collaborators. Global, regional, and national deaths, prevalence, disability-adjusted life years, and years lived with disability for chronic obstructive pulmonary disease and asthma, 1990-2015: a systematic analysis for the Global Burden of Disease Study 2015. Lancet Respir Med 2017; 5: 691-706. 
Atsou K, Chouaid C, Hejblum G. Variability of the chronic obstructive pulmonary disease key epidemiological data in Europe: systematic review. BMC Med 2011; 9: 7. Halbert RJ, Natoli JL, Gano A, et al. Global burden of COPD: systematic review and meta-analysis. Eur Respir J 2006; 28: 523-532.

Buist AS, McBurnie MA, Vollmer WM, et al. International variation in the prevalence of COPD (the BOLD Study): a population-based prevalence study. Lancet 2007; 370: 741-750.

6 Roche N, Dalmay F, Perez T, et al. Impact of chronic airflow obstruction in a working population. Eur Respir J 2008; 31: 1227-1233.

7 Menezes AMB, Perez-Padilla R, Jardim JR, et al. Chronic obstructive pulmonary disease in five Latin American cities (the PLATINO study): a prevalence study. Lancet 2005; 366: 1875-1881.

8 Scholes S, Moody A, Mindell JS. Estimating population prevalence of potential airflow obstruction using different spirometric criteria: a pooled cross-sectional analysis of persons aged 40-95 years in England and Wales. BMJ Open 2014; 4: e005685.

9 Beaglehole R, Bonita R, Alleyne G, et al. UN High-Level Meeting on Non-Communicable Diseases: addressing four questions. Lancet 2011; 378: 449-455.

10 Gershon AS, Warner L, Cascagnette P, et al. Lifetime risk of developing chronic obstructive pulmonary disease: a longitudinal population study. Lancet 2011; 378: 991-996.

11 Rabe KF, Watz H. Chronic obstructive pulmonary disease. Lancet 2017; 389: 1931-1940.

12 Adeloye D, Chua S, Lee C, et al. Global and regional estimates of COPD prevalence: Systematic review and meta-analysis. J Glob Health 2015; 5: 020415.

$13 \mathrm{Ng} \mathrm{M}$, Freeman MK, Fleming TD, et al. Smoking prevalence and cigarette consumption in 187 countries, 1980-2012. JAMA 2014; 311: 183-192.

14 Quach A, Giovannelli J, Chérot-Kornobis N, et al. Prevalence and underdiagnosis of airway obstruction among middle-aged adults in northern France: The ELISABET study 2011-2013. Respir Med 2015; 109: 1553-1561.

15 WHO. Projections of mortality and causes of death, 2016 to 2060. www.who.int/healthinfo/global_burden_ disease/projections/en/ Date last accessed: April 5, 2020; date last updated: April 2020.

16 The economic burden of lung disease. In: Gibson GJ, Loddenkemper R, Sibille Y, et al., eds. European Lung White Book. Sheffield, European Respiratory Society, 2013. www.erswhitebook.org/chapters/the-economicburden-of-lung-disease/

17 Rehman AU, Hassali MAA, Muhammad SA, et al. The economic burden of chronic obstructive pulmonary disease (COPD) in Europe: results from a systematic review of the literature. Eur J Health Econ 2020; 21: $181-194$.

18 Molinari N, Briand C, Vachier I, et al. Hospitalizations for COPD exacerbations: trends and determinants of death. COPD 2015; 12: 621-627.

19 Laurendeau C, Chouaid C, Roche N, et al. [Management and costs of chronic pulmonary obstructive disease in France in 2011]. Rev Mal Respir 2015; 32: 682-691.

20 Toy EL, Gallagher KF, Stanley EL, et al. The economic impact of exacerbations of chronic obstructive pulmonary disease and exacerbation definition: a review. COPD 2010; 7: 214-228.

21 Hilleman DE, Dewan N, Malesker M, et al. Pharmacoeconomic evaluation of COPD. Chest 2000; 118: $1278-1285$.

22 Mannino DM, Buist AS, Petty TL, et al. Lung function and mortality in the United States: data from the First National Health and Nutrition Examination Survey follow up study. Thorax 2003; 58: 388-393.

23 Lipson DA, Barnhart F, Brealey N, et al. Once-daily single-inhaler triple versus dual therapy in patients with COPD. N Engl J Med 2018; 378: 1671-1680.

24 Agusti A, Bel E, Thomas M, et al. Treatable traits: toward precision medicine of chronic airway diseases. Eur Respir J 2016; 47: 410-419.

Global Initiative for Chronic Obstructive Lung Disease. 2020 GOLD Reports - Global strategy for the diagnosis, management, and prevention of chronic obstructive pulmonary disease. www.goldcopd.org/gold-reports/ Date last accessed: 2020; date last updated: 2021.

26 Hogg JC. Pathophysiology of airflow limitation in chronic obstructive pulmonary disease. Lancet 2004; 364: 709-721.

27 Jones JG, Lawler P, Crawley JCW, et al. Increased alveolar epithelial permeability in cigarette smokers. Lancet 1980; 315: 66-68.

28 Agustí A, Hogg JC. Update on the pathogenesis of COPD. Reply. N Engl J Med 2019; 381: 2484.

29 McDonough JE, Yuan R, Suzuki M, et al. Small-airway obstruction and emphysema in chronic obstructive pulmonary disease. N Engl J Med 2011; 365: 1567-1575.

30 Salvi SS, Barnes PJ. Chronic obstructive pulmonary disease in non-smokers. Lancet 2009; 374: $733-743$.

31 Lelieveld J, Evans JS, Fnais M, et al. The contribution of outdoor air pollution sources to premature mortality on a global scale. Nature 2015; 525: 367-371.

32 Lange P, Celli B, Agustí A, et al. Lung-function trajectories leading to chronic obstructive pulmonary disease. N Engl J Med 2015; 373: 111-122

33 Martinez FD. Early-life origins of chronic obstructive pulmonary disease. N Engl J Med 2016; 375: 871-878.

34 Duffy S, Weir M, Criner GJ. The complex challenge of chronic obstructive pulmonary disease. Lancet Respir Med 2015; 3: 917-919.

35 Tobacco and Genetics Consortium. Genome-wide meta-analyses identify multiple loci associated with smoking behavior. Nat Genet 2010; 42: 441-447.

36 Sakornsakolpat P, Prokopenko D, Lamontagne M, et al. Genetic landscape of chronic obstructive pulmonary disease identifies heterogeneous cell-type and phenotype associations. Nat Genet 2019; 51: 494-505.

37 Soler Artigas M, Loth DW, Wain LV, et al. Genome-wide association and large-scale follow up identifies 16 new loci influencing lung function. Nat Genet 2011; 43: 1082-1090.

38 Hobbs BD, de Jong K, Lamontagne M, et al. Genetic loci associated with chronic obstructive pulmonary disease overlap with loci for lung function and pulmonary fibrosis. Nat Genet 2017; 49: 426-432. 
Bafadhel M, McKenna S, Terry S, et al. Acute exacerbations of chronic obstructive pulmonary disease identification of biologic clusters and their biomarkers. Am J Respir Crit Care Med 2011; 184: 662-671.

Narendra DK, Hanania NA. Targeting IL-5 in COPD. Int J Chron Obstruct Pulmon Dis 2019; 14: 1045-1051.

Barnes PJ. Cellular and molecular mechanisms of asthma and COPD. Clin Sci (Lond) 2017; 131: 1541-1558.

Capron T, Bourdin A, Perez T, et al. COPD beyond proximal bronchial obstruction: phenotyping and related tools at the bedside. Eur Respir Rev 2019; 28: 190010.

D'silva L, Hassan N, Wang HY, et al. Heterogeneity of bronchitis in airway diseases in tertiary care clinica practice. Can Respir J 2011; 18: 144-148.

Yousuf A, Brightling CE. Biologic drugs: a new target therapy in COPD? COPD 2018; 15: 99-107.

Sun J, Liu T, Yan Y, et al. The role of Th1/Th2 cytokines played in regulation of specific CD4+ Th1 cell conversion and activation during inflammatory reaction of chronic obstructive pulmonary disease. Scand $J$ Immunol 2018; 88: e12674.

Saha S, Brightling CE. Eosinophilic airway inflammation in COPD. Int J Chron Obstruct Pulmon Dis 2006; 1: $39-47$.

Denburg JA. Bone marrow in atopy and asthma: hematopoietic mechanisms in allergic inflammation. Immunol Today 1999; 20: 111-113.

Tashkin DP, Wechsler ME. Role of eosinophils in airway inflammation of chronic obstructive pulmonary disease. Int J Chron Obstruct Pulmon Dis 2018; 13: 335-349.

Nixon J, Newbold P, Mustelin T, et al. Monoclonal antibody therapy for the treatment of asthma and chronic obstructive pulmonary disease with eosinophilic inflammation. Pharmacol Ther 2017; 169: 57-77.

Hogan SP, Rosenberg HF, Moqbel R, et al. Eosinophils: biological properties and role in health and disease. Clin Exp Allergy 2008; 38: 709-750.

Yun JH, Lamb A, Chase R, et al. Blood eosinophil count thresholds and exacerbations in patients with chronic obstructive pulmonary disease. J Allergy Clin Immunol 2018; 141: 2037-2047.e10.

Farne HA, Wilson A, Powell C, et al. Anti-IL5 therapies for asthma. Cochrane Database Syst Rev 2017; 9: CD010834.

Brusselle GG, Maes T, Bracke KR. Eosinophils in the spotlight: Eosinophilic airway inflammation in nonallergic asthma. Nat Med 2013; 19: 977-979.

Spits H, Artis D, Colonna M, et al. Innate lymphoid cells: a proposal for uniform nomenclature. Nat Rev Immunol 2013; 13: 145-149.

Mkorombindo T, Dransfield MT. Mepolizumab in the treatment of eosinophilic chronic obstructive pulmonary disease. Int J Chron Obstruct Pulmon Dis 2019; 14: 1779-1787.

Han MK, Agusti A, Calverley PM, et al. Chronic obstructive pulmonary disease phenotypes: the future of COPD. Am J Respir Crit Care Med 2010; 182: 598-604.

Lange P, Halpin DM, O'Donnell DE, et al. Diagnosis, assessment, and phenotyping of COPD: beyond FEV Int J Chron Obstruct Pulmon Dis 2016; 11: 3-12.

Barnes PJ. Asthma-COPD overlap. Chest 2016; 149: 7-8.

Bourdin A, Suehs CM, Marin G, et al. Asthma, COPD, and overlap in a national cohort: ACO on a gradient. J Allergy Clin Immunol 2018; 141: 1516-1518.

Gibson PG, McDonald VM. Asthma-COPD overlap 2015: now we are six. Thorax 2015; 70: 683-691. J Respir Crit Care Med 1994; 150: 1646-1652.

Leigh R, Pizzichini MMM, Morris MM, et al. Stable COPD: predicting benefit from high-dose inhaled corticosteroid treatment. Eur Respir J 2006; 27: 964-971.

Singh D, Kolsum U, Brightling CE, et al. Eosinophilic inflammation in COPD: prevalence and clinical characteristics. Eur Respir J 2014; 44: 1697-1700.

Siva R, Green RH, Brightling CE, et al. Eosinophilic airway inflammation and exacerbations of COPD: a randomised controlled trial. Eur Respir J 2007; 29: 906-913.

Pascoe S, Locantore N, Dransfield MT, et al. Blood eosinophil counts, exacerbations, and response to the addition of inhaled fluticasone furoate to vilanterol in patients with chronic obstructive pulmonary disease: a secondary analysis of data from two parallel randomised controlled trials. Lancet Respir Med 2015; 3: 435-442. Agusti A, Fabbri LM, Singh D, et al. Inhaled corticosteroids in COPD: friend or foe? Eur Respir J 2018; 52: 1801219.

Liesker JJW, Bathoorn E, Postma DS, et al. Sputum inflammation predicts exacerbations after cessation of inhaled corticosteroids in COPD. Respir Med 2011; 105: 1853-1860.

Vedel-Krogh S, Nielsen SF, Lange P, et al. Blood eosinophils and exacerbations in chronic obstructive pulmonary disease. The Copenhagen General Population Study. Am J Respir Crit Care Med 2016; 193: 965-974.

Brightling CE, McKenna S, Hargadon B, et al. Sputum eosinophilia and the short term response to inhaled mometasone in chronic obstructive pulmonary disease. Thorax 2005; 60: 193-198.

Brightling CE, Monteiro W, Ward R, et al. Sputum eosinophilia and short-term response to prednisolone in chronic obstructive pulmonary disease: a randomised controlled trial. Lancet 2000; 356: 1480-1485.

Tine $\mathrm{M}$, Biondini $\mathrm{D}$, Semenzato $\mathrm{U}$, et al. Reassessing the role of eosinophils as a biomarker in chronic obstructive pulmonary disease. J Clin Med 2019; 8: 962.

George L, Taylor AR, Esteve-Codina A, et al. Blood eosinophil count and airway epithelial transcriptome relationships in COPD versus asthma. Allergy 2020; 75: 370-380.

Christenson SA, Steiling K, van den Berge M, et al. Asthma-COPD overlap. Clinical relevance of genomic signatures of type 2 inflammation in chronic obstructive pulmonary disease. Am J Respir Crit Care Med 2015 191: 758-766.

75 McLeod O, Silveira A, Valdes-Marquez E, et al. Genetic loci on chromosome 5 are associated with circulating levels of interleukin-5 and eosinophil count in a European population with high risk for cardiovascular disease. Cytokine 2016; 81: 1-9.

Nair P, Pizzichini MM, Kjarsgaard M, et al. Mepolizumab for prednisone-dependent asthma with sputum eosinophilia. N Engl J Med 2009; 360: 985-993. 
Haldar P, Brightling CE, Hargadon B, et al. Mepolizumab and exacerbations of refractory eosinophilic asthma. N Engl J Med 2009; 360: 973-984.

Pavord ID, Korn S, Howarth P, et al. Mepolizumab for severe eosinophilic asthma (DREAM): a multicentre, double-blind, placebo-controlled trial. Lancet 2012; 380: 651-659.

Ortega HG, Liu MC, Pavord ID, et al. Mepolizumab treatment in patients with severe eosinophilic asthma. N Engl J Med 2014; 371: 1198-1207.

Wang JM, Rambaldi A, Biondi A, et al. Recombinant human interleukin 5 is a selective eosinophil chemoattractant. Eur J Immunol 1989; 19: 701-705.

Jatakanon A, Lim S, Barnes PJ. Changes in sputum eosinophils predict loss of asthma control. Am J Respir Crit Care Med 2000; 161: 64-72.

Possa SS, Leick EA, Prado CM, et al. Eosinophilic inflammation in allergic asthma. Front Pharmacol 2013; 4: 46. Fala L. Nucala (Mepolizumab): First IL-5 Antagonist Monoclonal Antibody FDA Approved for Maintenance Treatment of Patients with Severe Asthma. Am Health Drug Benefits 2016; 9: 106-110.

FDA, Clinical Review - NUCALA (mepolizumab). www.fda.gov/media/95114/download. Date last accessed: 2020; date last updated: 2015.

Hart TK, Cook RM, Zia-Amirhosseini P, et al. Preclinical efficacy and safety of mepolizumab (SB-240563), a humanized monoclonal antibody to IL-5, in cynomolgus monkeys. J Allergy Clin Immunol 2001; 108: 250-257.

Smith DA, Minthorn EA, Beerahee M. Pharmacokinetics and pharmacodynamics of mepolizumab, an anti-interleukin-5 monoclonal antibody. Clin Pharmacokinet 2011; 50: 215-227.

EMA, Nucala: Summary Of Product Characteristics. www.ema.europa.eu/en/documents/product-information/ nucala-epar-product-information_en.pdf. Date last accessed: 2020; date last updated: 2015.

Ortega HG, Yancey SW, Mayer B, et al. Severe eosinophilic asthma treated with mepolizumab stratified by baseline eosinophil thresholds: a secondary analysis of the DREAM and MENSA studies. Lancet Respir Med 2016; 4: 549-556.

Powell C, Milan SJ, Dwan K, et al. Mepolizumab versus placebo for asthma. Cochrane Database Syst Rev 2015; 7: CD010834.

Dasgupta A, Kjarsgaard M, Capaldi D, et al. A pilot randomised clinical trial of mepolizumab in COPD with eosinophilic bronchitis. Eur Respir J 2017; 49: 1602486.

Pavord ID. Biologics and chronic obstructive pulmonary disease. J Allergy Clin Immunol 2018; 141: 1983-1991. Shahlav-Monavvar P, Mobasher-Jannat A. Mepolizumab for eosinophilic COPD. N Engl J Med 2018; 378: 681.

Xia Y, Li W, Shen H. Mepolizumab for eosinophilic COPD. N Engl J Med 2018; 378: 680-681.

Pavord ID, Chanez P, Criner GJ, et al. Mepolizumab for eosinophilic chronic obstructive pulmonary disease. N Engl J Med 2017; 377: 1613-1629.

Ortega H, Lemiere C, Llanos JP, et al. Outcomes following mepolizumab treatment discontinuation: real-world experience from an open-label trial. Allergy Asthma Clin Immunol 2019; 15: 37.

Haldar P, Brightling CE, Singapuri A, et al. Outcomes after cessation of mepolizumab therapy in severe eosinophilic asthma: a 12-month follow-up analysis. J Allergy Clin Immunol 2014; 133: 921-923.

Moore WC, Kornmann O, Humbert M, et al. Outcomes following continuation or stopping long-term mepolizumab treatment in patients with severe eosinophilic asthma: the Randomized Comet Trial. Am J Respir Crit Care Med 2020; 201: A4211.

Condreay LD, Gao C, Bradford E, et al. Yancey, genetic associations with mepolizumab efficacy in COPD with peripheral blood eosinophilia. Respir Med 2019; 155: 26-28.

EMA, CINQAERO: Summary Of Product Characteristics. www.ema.europa.eu/en/documents/product-information/ cinqaero-epar-product-information_en.pdf Date last accessed: 2020; date last updated: 2016.

Kips JC, O'Connor BJ, Langley SJ, et al. Effect of SCH55700, a humanized anti-human interleukin-5 antibody, in severe persistent asthma: a pilot study. Am J Respir Crit Care Med 2003; 167: 1655-1659.

Castro M, Mathur S, Hargreave F, et al. Reslizumab for poorly controlled, eosinophilic asthma: a randomized, placebo-controlled study. Am J Respir Crit Care Med 2011; 184: 1125-1132.

Ghazi A, Trikha A, Calhoun WJ. Benralizumab: a humanized mAb to IL-5R $\alpha$ with enhanced antibody-dependent cell-mediated cytotoxicity--a novel approach for the treatment of asthma. Expert Opin Bio Ther 2012; 12: 113-118.

Kolbeck R, Kozhich A, Koike M, et al. MEDI-563, a humanized anti-IL-5 receptor alpha mAb with enhanced antibody-dependent cell-mediated cytotoxicity function. J Allergy Clin Immunol 2010; 125: 1344-1353.

Pham T-H, Damera G, Newbold P, et al. Reductions in eosinophil biomarkers by benralizumab in patients with asthma. Respir Med 2016; 111: 21-29.

Tan LD, Bratt JM, Godor D, et al. Benralizumab: a unique IL-5 inhibitor for severe asthma. J Asthma Allergy 2016; 9: 71-81.

Laviolette M, Gossage DL, Gauvreau G, et al. Effects of benralizumab on airway eosinophils in asthmatic patients with sputum eosinophilia. J Allergy Clin Immunol 2013; 132: 1086-1096.

Bleecker ER, FitzGerald JM, Chanez P, et al. Efficacy and safety of benralizumab for patients with severe asthma uncontrolled with high-dosage inhaled corticosteroids and long-acting $\beta 2$-agonists (SIROCCO): a randomised, multicentre, placebo-controlled phase 3 trial. Lancet 2016; 388: 2115-2127.

FitzGerald JM, Bleecker ER, Nair P, et al. Benralizumab, an anti-interleukin-5 receptor $\alpha$ monoclonal antibody, as add-on treatment for patients with severe, uncontrolled, eosinophilic asthma (CALIMA): a randomised, double-blind, placebo-controlled phase 3 trial. Lancet 2016; 388: 2128-2141.

Bourdin A, Shaw D, Menzies-Gow A, et al. Two-year integrated steroid-sparing analysis and safety of benralizumab for severe asthma. J Asthma 2021; 58: 514-522.

Castro M, Wenzel SE, Bleecker ER, et al. Benralizumab, an anti-interleukin 5 receptor $\alpha$ monoclonal antibody, versus placebo for uncontrolled eosinophilic asthma: a phase $2 \mathrm{~b}$ randomised dose-ranging study. Lancet Respir Med 2014; 2: 879-890. sputum eosinophilia: a randomised, double-blind, placebo-controlled, phase 2a study. Lancet Respir Med 2014; 2 : 891-901. 
Criner GJ, Celli BR, Brightling CE, et al. Benralizumab for the Prevention of COPD Exacerbations. N Engl J Med 2019; 381: 1023-1034.

113 Criner GJ, Celli BR, Singh D, et al. Predicting response to benralizumab in chronic obstructive pulmonary disease: analyses of GALATHEA and TERRANOVA studies. Lancet Respir Med 2020; 8: 158-170.

114 May RD, Fung M. Strategies targeting the IL-4/IL-13 axes in disease. Cytokine 2015; 75: 89-116.

115 Brightling CE, Saha S, Hollins F. Interleukin-13: prospects for new treatments. Clin Exp Allergy 2010; 40: 42-49.

116 Kaur D, Hollins F, Woodman L, et al. Mast cells express IL-13R alpha 1: IL-13 promotes human lung mast cell proliferation and Fc epsilon RI expression. Allergy 2006; 61: 1047-1053.

117 Howard TD, Koppelman GH, Xu J, et al. Gene-gene interaction in asthma: IL4RA and IL13 in a Dutch population with asthma. Am J Hum Genet 2002; 70: 230-236.

118 Marone G, Granata F, Pucino V, et al. The intriguing role of interleukin 13 in the pathophysiology of asthma. Front Pharmacol 2019; 10: 1387.

119 Panettieri RA, Wang M, Braddock M, et al. Tralokinumab for the treatment of severe, uncontrolled asthma: the ATMOSPHERE clinical development program. Immunotherapy 2018; 10: 473-490.

120 Wang I-M, Lin H, Goldman SJ, et al. STAT-1 is activated by IL-4 and IL-13 in multiple cell types. Mol Immunol 2004; 41: 873-884

121 Yang SJ, Allahverdian S, Saunders ADR, et al. IL-13 signaling through IL-13 receptor $\alpha 2$ mediates airway epithelial wound repair. FASEB J 2019; 33: 3746-3757.

122 White SR, Martin LD, Stern R, et al. Expression of IL-4/IL-13 receptors in differentiating human airway epithelial cells. Am J Physiol Lung Cell Mol Physiol 2010; 299: L681-L693.

123 Hilton DJ, Zhang JG, Metcalf D, et al. Cloning and characterization of a binding subunit of the interleukin 13 receptor that is also a component of the interleukin 4 receptor. Proc Natl Acad Sci USA 1996; 93: 497-501.

124 van der Pouw Kraan TCTM, Küçükaycan M, Bakker AM, et al. Chronic obstructive pulmonary disease is associated with the -1055 IL-13 promoter polymorphism. Genes Immun 2002; 3: 436-439.

125 Grünig G, Warnock M, Wakil AE, et al. Requirement for IL-13 independently of IL-4 in experimental asthma. Science 1998; 282: 2261-2263.

126 Blanchard C, Mishra A, Saito-Akei H, et al. Inhibition of human interleukin-13-induced respiratory and oesophageal inflammation by anti-human-interleukin-13 antibody (CAT-354). Clin Exp Allergy 2005; 35: 1096-1103.

127 Schmid-Grendelmeier P, Altznauer F, Fischer B, et al. Eosinophils express functional IL-13 in eosinophilic inflammatory diseases. J Immunol 2002; 169: 1021-1027.

128 Jiang M, Liu H, Li Z, et al. ILC2 s induce adaptive Th2-type immunity in acute exacerbation of chronic obstructive pulmonary disease. Mediators Inflamm 2019; 2019: 3140183.

129 Bel EH, Ten Brinke A. new anti-eosinophil drugs for asthma and COPD: targeting the trait! Chest 2017; 152: 1276-1282.

130 Wenzel S, Castro M, Corren J, et al. Dupilumab efficacy and safety in adults with uncontrolled persistent asthma despite use of medium-to-high-dose inhaled corticosteroids plus a long-acting $\beta_{2}$ agonist: a randomised double-blind placebo-controlled pivotal phase $2 \mathrm{~b}$ dose-ranging trial. Lancet 2016; 388: 31-44.

131 Hanania NA, Noonan M, Corren J, et al. Lebrikizumab in moderate-to-severe asthma: pooled data from two randomised placebo-controlled studies. Thorax 2015; 70: 748-756.

132 Piper E, Brightling C, Niven R, et al. A phase II placebo-controlled study of tralokinumab in moderate-to-severe asthma. Eur Respir J 2013; 41: 330-338.

133 Panettieri RA, Sjöbring U, Péerffy A, et al. Tralokinumab for severe, uncontrolled asthma (STRATOS 1 and STRATOS 2): two randomised, double-blind, placebo-controlled, phase 3 clinical trials. Lancet Respir Med 2018; 6: 511-525.

134 Marone G, Spadaro G, Braile M, et al. Tezepelumab: a novel biological therapy for the treatment of severe uncontrolled asthma. Expert Opin Investig Drugs 2019; 28: 931-940.

135 Soumelis V, Reche PA, Kanzler H, et al. Human epithelial cells trigger dendritic cell mediated allergic inflammation by producing TSLP. Nat Immunol 2002; 3: 673-680.

136 Corren J, Parnes JR, Wang L, et al. Tezepelumab in adults with uncontrolled asthma. N Engl J Med 2017; 377: 936-946.

137 Djukanović R, Wilson SJ, Kraft M, et al. Effects of treatment with anti-immunoglobulin E antibody omalizumab on airway inflammation in allergic asthma. Am J Respir Crit Care Med 2004; 170: 583-593.

138 Thomson NC, Chaudhuri R. Omalizumab: clinical use for the management of asthma. Clin Med Insights Circ Respir Pulm Med 2012; 6: 27-40.

139 Takaku Y, Soma T, Nishihara F, et al. Omalizumab attenuates airway inflammation and interleukin-5 production by mononuclear cells in patients with severe allergic asthma. Int Arch Allergy Immunol 2013; 161: Suppl. 2, 107-117.

140 Maltby S, Gibson PG, Powell H, et al. Omalizumab treatment response in a population with severe allergic asthma and overlapping COPD. Chest 2017; 151: 78-89.

141 Hanania NA, Chipps BE, Griffin NM, et al. Omalizumab effectiveness in asthma-COPD overlap: post hoc analysis of PROSPERO. J Allergy Clin Immunol 2019; 143: 1629-1633.

142 Taillé C, Chanez P, Devouassoux G, et al. Mepolizumab in a population with severe eosinophilic asthma and corticosteroid dependence: results from a French early access programme. Eur Respir J 2020; 55: 1902345.

143 Eltboli O, Bafadhel M, Hollins F, et al. COPD exacerbation severity and frequency is associated with impaired macrophage efferocytosis of eosinophils. BMC Pulm Med 2014; 14: 112.

144 Singh D, Bafadhel M, Brightling CE, et al. Blood eosinophil counts in clinical trials for chronic obstructive pulmonary disease. Am J Respir Crit Care Med 2020; 202: 660-671.

145 Salter BM, Sehmi R. Hematopoietic processes in eosinophilic asthma. Chest 2017; 152: 410-416.

146 Kato Y, Takabayashi T, Sakashita M, et al. Expression and functional analysis of CST1 in intractable nasal polyps. Am J Respir Cell Mol Biol 2018; 59: 448-457.

147 Castro M, Corren J, Pavord ID, et al. Dupilumab efficacy and safety in moderate-to-severe uncontrolled asthma. N Engl J Med 2018; 378: 2486-2496. 
148 Rabe KF, Nair P, Brusselle G, et al. Efficacy and safety of dupilumab in glucocorticoid-dependent severe asthma. N Engl J Med 2018; 378: 2475-2485.

149 Castro M, Rabe KF, Corren J, et al. Dupilumab improves lung function in patients with uncontrolled, moderate-to-severe asthma. ERJ Open Res 2020; 6: 00204-2019.

150 Doyle AD, Mukherjee M, LeSuer WE, et al. Eosinophil-derived IL-13 promotes emphysema. Eur Respir J 2019; 53: 1801291.

151 Higham A, Beech A, Wolosianka S, et al. Type 2 Inflammation in Eosinophilic Chronic Obstructive Pulmonary Disease. Allergy 2020; in press [https://doi.org/10.1111/all.14661]. 